\title{
Asymmetric state feedback for linear plants with asymmetric input saturation
}

\author{
S. Mariano, F. Blanchini, Senior Member, IEEE, S. Formentin, Member, IEEE, L. Zaccarian, Fellow, IEEE
}

\begin{abstract}
We consider a linear plant with decentralized input saturation, whose limits are not necessarily symmetric. We propose a nonlinear static state feedback stabilizer that is asymmetric, in such a way that the non-symmetric nature of the saturation is fully exploited in the control design, for larger regions of attraction. We show by example that the proposed technique provides significantly larger regions of attractions as compared to the symmetric solution results, in a case where the positive and negative saturation limits are different.

Index Terms - Constrained control; Lyapunov methods; Stability of nonlinear systems
\end{abstract}

\section{INTRODUCTION AND BACKGROUND}

Most existing works dealing with the design of a state feedback (locally or globally) stabilizing the origin of a linear system with input saturation address the simplified problem of symmetric saturation (see, e.g., the extensive surveys in the books [9], [21], [17]). Nevertheless, in many practical situations (see, e.g., the applications in [18], [20]), the two limits are substantially different and the typical solution adopted in the literature is to disregard achievable control performance by conservatively focusing on the smallest limit.

While the majority of the existing results deals with the symmetric saturated case, a few papers have been published addressing the nonsymmetric case more directly. In particular, several continuous-time and discrete-time techniques have been proposed in [1] and references therein, based on the development of non-symmetric Lyapunov functions in the presence of linear (symmetric) stabilizers with nonsymmetric saturation (these, in turn, being developments of the pioneering ideas reported in [2]). Similar approaches have been followed in [10], [11], even though an informative study is carried out therein illustrating how stretched the nullcontrollability set is, whenever the input saturation limits are unbalanced (this being a main motivation for the approach adopted here). Another line of research relies on shifting the coordinate system in such a way that the saturation becomes symmetric, but the drawback of this is that the point being stabilized is not anymore the origin (see the

Research supported in part by the Agence Nationale de la Recherche (ANR) via grant Hybrid And Networked Dynamical sYstems (HANDY), number ANR-18-CE40-0010.

Simone Mariano is with Université de Lorraine, CNRS, CRAN, F-54000 Nancy, France. Franco Blanchini is with Dipartimento di Matematica e Informatica, University of Udine, Via Delle Scienze 208, Udine, Italy. Simone Formentin is with Dipartimento di Elettronica, Informazione e Bioingegneria, Politecnico di Milano, Piazza Leonardo da Vinci 32, 20133 Milano, Italy. Luca Zaccarian is with CNRS, LAAS, 7 avenue du colonel Roche, F-31400 Toulouse, France, Univ. de Toulouse, LAAS, F-31400 Toulouse, France, and Dip. di Ingegneria Industriale, University of Trento, Italy. recent book [3, ch. 8] and references therein). Perhaps the most sophisticated solution to the asymmetric bounded stabilization problem is given in [19], where the authors propose a switching dynamical control scheme, designed by solving suitable linear matrix inequalities and capable of exploiting the available range of the control action on both sides of the saturation levels. A parallel approach has been proposed in [13] and [8], addressing respectively continuous-time and discrete-time systems, where suitable LMIs addressing each set in the partition ensure desirable properties of a piecewise quadratic Lyapunov function with symmetric stabilizing saturated linear feedbacks.

Different from the above mentioned approaches, inspired by the stretched shapes of the null controllability regions reported in [10], [11], the control design technique proposed in this paper corresponds to a nonlinear asymmetric stretch of a symmetric control law, which is capable of providing enlarged certified stability regions (subsets of the domain of attraction), as compared to the symmetric counterpart. The core intuition behind the proposed controller is to address the control design in shifted state coordinates under which the saturation limits would be symmetric, and then once the dynamics is analysed in that symmetric shifted setting, use the non-symmetric solution of [4] for stabilizing the origin (the origin, in those coordinates, corresponds to a shifted, off-center equilibrium).

\section{SyMmetriC STABILIZER}

Consider the linear saturated continuous-time plant:

$$
\dot{x}=A x+B \operatorname{sat}(u),
$$

where $x \in \mathbb{R}^{n}$ is the plant state, $u \in \mathbb{R}^{m}$ is the unconstrained plant input, $A \in \mathbb{R}^{n \times n}, B \in \mathbb{R}^{n \times m}$ and sat denotes the standard vector saturation having components $\operatorname{sat}_{i}\left(u_{i}\right)=$ $\max \left(\min \left(u_{i}^{+}, u_{i}\right),-u_{i}^{-}\right)$, being $u_{i}^{+}>0$ and $u_{i}^{-}>0$ the upper and lower bounds, respectively, on input channel $i$. Also denote by $u^{+}$and $u^{-}$, respectively, the vectors obtained by stacking together the elements $u_{i}^{+}$and $u_{i}^{-}$, respectively, for all $i=1, \ldots, m$. To suitably stabilize the origin of (1), a standard assumption is that the origin is in the interior of the subset of $\mathbb{R}^{m}$ where the input is not saturated (namely the set where $u=\operatorname{sat}(u)$ ). Such an assumption, formalized below, is generally necessary to achieve local exponential stabilization of (1) when $A$ is not Hurwitz (unless redundant input directions are available).

Assumption 1: All the elements of vectors $u^{+}$and $u^{-}$are positive, that is, $u^{+}>0$ and $u^{-}>0$, where the inequalities should be understood componentwise. 
Asymmetric saturation limits may emerge due to shifted coordinate systems with constant setpoints, or simply due to specific actuators with asymmetric stroke. For the proposed paradigm to make sense, we assume the following.

Assumption 2: Matrix $A$ is non-singular.

Under the assumption that $x$ be accessible for measurement, a nonlinear state-feedback controller of the form

$$
u=K x+L \mathrm{dz}(u),
$$

can be designed based on the plant dynamics, in order to induce suitable closed-loop properties when interconnected to the plant (1). In (2), dz denotes the deadzone function, defined as $\operatorname{dz}(u):=u-\operatorname{sat}(u)$. The resulting nonlinear control system obtained by lumping together (1) and (2) can be compactly written as

$$
\begin{aligned}
\dot{x} & =(A+B K) x-(B-B L) \mathrm{dz}(u) \\
u & =K x+L \mathrm{dz}(u) .
\end{aligned}
$$

We call (2) symmetric stabilizer because in a neighbourhood of the origin in dynamics (3) is symmetric. In the case where the state $x$ is not accessible the state feedback solution (2) can be replaced by a dynamic output feedback solution with anti-windup, such as in [6]. Extending our state-feedback approach to that case is an interesting future direction. The control parameters $K \in \mathbb{R}^{m \times n}$ and $L \in \mathbb{R}^{m \times m}$ can be designed according to several different criteria. In this paper, we address the problem of simultaneous stabilization and maximization of a quadratic estimate of the Basin of Attraction of the origin for (3). To this end, a slight extension (mentioned in the proof below) of the classical results proven in [5], [9], [12] and [17, §3.2.1] allow to state the following theorem.

Theorem 1 (Symmetric stabilizer): Given plant (1), define the lowest limits $\underline{u}:=\min \left(u^{+}, u^{-}\right)$, where the minimum should be computed componentwise. Then, given any solution to the following convex optimization:

$$
\begin{gathered}
\underset{\substack{Q \in \mathbb{R}^{n \times n}, W, Y \in \mathbb{R}^{m \times n} \\
U, X \in \mathbb{R}^{m \times m}}}{\log \operatorname{det}(Q) \text { subject to: }} \\
Q=Q^{T}>0, U>0 \text { diagonal } \\
\operatorname{He}\left[\begin{array}{cc}
A Q+B W & -B U+B X \\
W+Y & X-U
\end{array}\right]<0 \\
\\
{\left[\begin{array}{cc}
\underline{u}_{k}^{2} & Y_{[k]} \\
Y_{[k]}^{T} & Q
\end{array}\right] \geq 0, \quad k=1, \ldots, m}
\end{gathered}
$$

where $\operatorname{He}(Z)=Z+Z^{T}, Y_{[k]}$ denotes the $k$-th row of matrix $Y$ and $\underline{u}_{k}$ denotes the $k$-the entry of vector $\underline{u}$, by selecting

$$
K=W Q^{-1}, \quad L=X U^{-1}, \quad P=Q^{-1},
$$

the nonlinear algebraic loop in (2) is well posed (in the sense that its solution is unique and Lipschitz) and the origin is locally exponentially stable, with basin of attraction containing the set:

$$
\mathcal{E}_{s}(P):=\left\{x: x^{T} P x \leq 1\right\} .
$$

Proof: With symmetric saturation limits, that is $\breve{u}=$ $u^{+}=u^{-}$, the proof follows the generalized (local) sector condition approach of [5], [9], [12]. However, the same exact proofs also apply to the case $\underline{u} \leq u^{+}$and $\underline{u} \leq u^{-}$, which holds from the definition of $\underline{u}$.

The above result represents a computationally efficient way to design a stabilizing controller which also maximizes the basin of attraction for the closed loop (3), and (4) is feasible (see, e.g., [12]) for any stabilizable pair $(A, B)$ under Assumption 1. However, the guaranteed estimate can be quite conservative when the upper and lower limits of the inputs are different, namely when $u_{k}^{+} \neq u_{k}^{-}$for some $k$.

\section{ASYMMETRIC STABILIZER}

Let us define the average saturation range $\bar{u}$ and the average saturation center $u_{\circ}$ as:

$$
\begin{aligned}
\bar{u} & :=\left(u^{+}+u^{-}\right) / 2, \\
u_{\circ} & :=\left(u^{+}-u^{-}\right) / 2 .
\end{aligned}
$$

Due to Assumption 2, we may introduce the mid-range point $x_{\circ} \in \mathbb{R}^{n}$ satisfying

$$
x_{\circ}:=-A^{-1} B u_{\circ}, \quad A x_{\circ}+B u_{\circ}=0 .
$$

In particular, $x_{\circ}$ characterizes an equilibrium point induced by input $u_{\circ}$. For $(A, B)$ to be stabilizable it is necessary that $\operatorname{rank}[A B]=n$ (from the $\mathrm{PBH}$ test applied to the zero eigenvalue). When $u_{\circ} \neq 0$ (as customary in the asymmetric case $\left.u^{+} \neq u^{-}\right), x_{\circ}$ satisfying the second equation in (8) exists only if $\operatorname{rank}[A]=\operatorname{rank}[A B]=n$, which reveals that Assumption 2 is necessary (and sufficient) under a stabilizability condition.

Based on the quantities introduced above we propose an alternative nonlinear (and asymmetric) stabilizer based on a scalar $\lambda \in[0,1]$, whose expression generalizes the one of Theorem 1 (which becomes a special case when $\lambda=0$ ), having the following form

$$
\begin{aligned}
& u=\kappa(x):= \\
& \left\{\begin{array}{lr}
K x+\gamma(x)\left(\lambda \delta_{\circ}+(L-I) \mathrm{dz}\left(\nu(x)+\lambda \delta_{\circ}\right)\right), & \text { if } x \neq 0, \\
K x, & \text { if } x=0,
\end{array}\right.
\end{aligned}
$$

where $\delta_{\circ}:=u_{\circ}-K x_{\circ}$, the function $\gamma$ is defined in (9b) at the top of the next page, and the function $\nu$ is the solution of the following nonlinear algebraic loop:

$$
\nu=\frac{K x}{\gamma(x)}+L \mathrm{dz}\left(\nu+\lambda \delta_{\circ}\right) .
$$

Remark 1: The algebraic loop appearing in (9c) can be eliminated by imposing $X=0$ in the matrix inequalities (4), which results in $L=0$. In that case we get $u=K x$ in (3) and $\nu=\frac{K x}{\gamma(x)}$, which in turns implies that function $\kappa$ in (9a) reduces to

$\kappa(x)= \begin{cases}K x+\gamma(x)\left(\lambda \delta_{\circ}-\mathrm{dz}\left(\frac{K x}{\gamma(x)}+\lambda \delta_{\circ}\right)\right), & \text { if } x \neq 0, \\ K x, & \text { if } x=0,\end{cases}$

corresponding to an explicit nonlinear state feedback that is easier to implement in real-time applications. For the case where $X \neq 0$ (and then $L \neq 0$ ), the algebraic loop (9c) (equivalently, the one in (2)) can be addressed using the 


$$
\gamma(x):=\left\{\min \left\{1,\left(1-\lambda\left|x_{\circ}\right|_{P}\right)^{-1}\left(-\frac{x_{\circ}^{T} P x}{\left|x_{\circ}\right|_{P}}+\sqrt{\left(1-\lambda\left|x_{\circ}\right|_{P}\right) \frac{|x|_{P}^{2}}{\lambda\left|x_{\circ}\right|_{P}}+\left(\frac{x_{\circ}^{T} P x}{\left|x_{\circ}\right|_{P}}\right)^{2}}\right)\right\}, \begin{array}{cl}
\text { if } \lambda x_{\circ} \neq 0, \\
1, & \text { if } \lambda x_{\circ}=0,
\end{array}\right.
$$

methods in [21, Sec. 2.3.7] or the equivalent QP optimization discussed in [16]. We emphasize that the algebraic loop is especially useful for the multi-input case $m>1$, see, e.g., [15]. For the case $m=1$ any nonzero selection of scalars $(X, U) \neq(0,0)$ in (4) can be exchanged for the alternative choice $(\bar{X}, \bar{U})=(0, U-X)$, thus removing algebraic loop and getting the same performance. This is clearly not possible when $m>1$ because $U$ must be diagonal.

The following lemma establishes a few useful properties of the proposed asymmetric stabilizer (9).

Lemma 1: If $\lambda^{2} x_{\circ}^{T} P x_{\circ}<1$ and $K$ and $L$ arise from the construction in Theorem 1, then the following holds:

1) function $\gamma$ in (9b) is locally bounded, continuous and locally homogeneous of degree one, in particular, for each $x \in \mathbb{R}^{n}$ and each scalar $s \geq 1, \gamma(s x)=$ $\min \{1, s \gamma(x)\}$

2) for each $x \neq 0$, the algebraic loop in (9c) is well posed (it has a unique and Lipschitz solution $x \mapsto \nu(x)$ );

3) function $\kappa$ in (9a) is continuous.

Proof: Item 1. Under the stated assumption, $\lambda\left|x_{\circ}\right|_{P}:=$ $\lambda \sqrt{x_{\circ}^{T} P x_{\circ}}<1$, therefore all the terms in (9b) are bounded for each value of $x$. Local homogeneity and the consequent continuity is easily verified by inspection.

Item 2. If $x \neq 0$, the algebraic loop in (9c) becomes:

$$
\nu+\lambda \delta_{\circ}=\frac{K x}{\gamma(x)}+\lambda \delta_{\circ}+L \mathrm{dz}\left(\nu+\lambda \delta_{\circ}\right),
$$

which coincides with the algebraic loop in (3) (with $u=\nu+$ $\lambda \delta_{\circ}$ and $K x$ replaced by the external input $\left.\frac{K x}{\gamma(x)}+\lambda \delta_{\circ}\right)$. The well-posedness of this nonlinear algebraic loop is established in Theorem 1.

Item 3. The continuity property of $\kappa$ away from the origin immediately follows from the fact that it is a composition of Lipschitz functions. At the origin, first note that when $\lambda x_{\circ}=$ 0 the stabilizer coincides with the symmetric one of Theorem 1 and continuity follows from well-posedness. When $\lambda x_{\circ} \neq 0$, close to the origin we have $\gamma(x)=|x| \gamma\left(\frac{x}{|x|}\right)$, meaning that the explicit solution $\nu(x)$ is locally constant around rays close to the origin. Therefore $\nu$ is uniformly upper bounded around the origin and the multiplicative $\gamma(x)$ in (9a), together with the fact that $\lim _{x \rightarrow 0} \gamma(x)=0$, guarantees continuity of $\kappa$ at the origin.

Starting from the above statements, we derive the next theorem, the main contribution of this paper.

Theorem 2 (Asymmetric stabilizer): Consider plant (1) and the average saturation center $u_{\circ}$ in (7b). Take any solution to the convex optimization (4a)-(4c) augmented with $\left[\begin{array}{cc}\mu & \mu x_{\circ}^{T} \\ \mu x_{\circ} & Q\end{array}\right]>0,\left[\begin{array}{cc}\underline{u}_{k}^{2}+\mu\left|u_{\circ, k}\right|\left(2 u_{k}+\left|u_{\circ, k}\right|\right) & Y_{[k]} \\ Y_{[k]}^{T} & Q\end{array}\right] \geq 0$, $k=1, \ldots, m$, and the extra decision variable $\mu \in[0,1]$. Then the asymmetric nonlinear control law (9) with selection $\lambda=\sqrt{\mu}$ and (5) is globally well defined and locally exponentially stabilizes the origin with basin of attraction containing the neighbourhood of the origin:

$$
\mathcal{E}_{a}\left(P, \lambda x_{\circ}\right):=\left\{x:\left(x-\lambda x_{\circ}\right)^{T} P\left(x-\lambda x_{\circ}\right) \leq 1\right\} .
$$

Proof: Using a congruence transformation in the left inequality and $\mu \leq \lambda^{2} \leq \lambda$ in the right inequality, constraints (14) imply

$$
\left[\begin{array}{cc}
1 & \lambda x_{\circ}^{T} \\
\lambda x_{\circ} & Q
\end{array}\right]>0,\left[\begin{array}{cc}
\left(\underline{u}_{k}+\lambda\left|u_{\circ, k}\right|\right)^{2} & Y_{[k]} \\
Y_{[k]}^{T} & Q
\end{array}\right] \geq 0,
$$

$k=1, \ldots, m$. The left inequality of (14) can be rearranged via a Schur complement to show that $\lambda^{2} x_{\circ}^{T} P x_{\circ}<1$, so that Lemma 1 applies and the feedback selection satisfies suitable regularity conditions.

Consider now the change of coordinates

$$
\begin{aligned}
& \tilde{x}=x-\lambda x_{\circ}, \\
& \tilde{u}=u-\lambda u_{\circ} .
\end{aligned}
$$

The plant dynamics (1) in the reference framework defined in (15) can be rewritten as

$$
\begin{aligned}
\dot{\tilde{x}} & =A x+B \operatorname{sat}(u)-\left(A \lambda x_{\circ}+B \lambda u_{\circ}\right) \\
& =A \tilde{x}+B \operatorname{sat}(u)-B \lambda u_{\circ}=A \tilde{x}+B \sigma(\tilde{u}),
\end{aligned}
$$

where $\sigma(\tilde{u}):=\operatorname{sat}\left(\tilde{u}+\lambda u_{\circ}\right)-\lambda u_{\circ}$ is a vector saturation function having lower and upper limits $u_{\sigma}^{-}:=u^{-}+\lambda u_{\circ}$ and $u_{\sigma}^{+}:=u^{+}-\lambda u_{\circ}$, respectively, whose components satisfy $\underline{u}_{k}+\lambda\left|u_{\circ, k}\right| \leq \min \left\{u_{\sigma, k}^{-}, u_{\sigma, k}^{+}\right\}$(in particular, from (7a), with $\lambda=1$ we recover the symmetric case $\left.u_{\sigma}^{-}=u_{\sigma}^{+}=\bar{u}\right)$.

Since any solution to (4a)-(4c), (12) satisfies (4) with (4d) replaced by the right inequalities in (14), then Theorem 1 guarantees that $K$ and $L$ selected as in (5) are such that the following algebraic loop is well posed:

$$
v=K z+L \mathrm{dz}_{\sigma}(v)
$$

where $\mathrm{dz}_{\sigma}(v):=v-\sigma(v)$. Moreover, introducing the feedback function

$$
\begin{aligned}
\phi(z) & :=\sigma(v)=v-\mathrm{dz}_{\sigma}(v) \\
& =K z+(L-I) \mathrm{dz}_{\sigma}(v),
\end{aligned}
$$

the feedback stabilizer $\tilde{u}=\phi(\tilde{x})$ stabilizes the origin $\tilde{x}=0$ of (16) with basin of attraction including the set

$$
\tilde{\mathcal{E}}_{s}(P):=\left\{\tilde{x}: \tilde{x}^{T} P \tilde{x} \leq 1\right\}=\mathcal{E}_{a}\left(P, \lambda x_{\circ}\right) .
$$

In the rest of the proof, we use the following result, which is a generalization of [4, Thm 3.3] with the explicit expression for ellipsoidal domains given in $[4, \S 6.1]$. 
Lemma 2: [4] Consider plant (16) and any point $x^{*} \in$ $\tilde{\mathcal{E}}_{s}(P)$ satisfying $A x^{*}+B u^{*}=0$ for some suitable vector $u^{*}$. Then the input selection

$$
\begin{aligned}
& \tilde{u}=u^{*}+\Psi\left(\tilde{x}, x^{*}\right)\left(\phi\left(x^{*}+\frac{\tilde{x}-x^{*}}{\Psi\left(\tilde{x}, x^{*}\right)}\right)-u^{*}\right) \\
& \Psi\left(\tilde{x}, x^{*}\right):=\frac{\frac{x^{* T} P \xi}{\left|x^{*}\right|_{P}}+\sqrt{\left(\frac{x^{* T} P \xi}{\left|x^{*}\right|_{P}}\right)^{2}+w \frac{\xi^{T} P \xi}{\left|x^{*}\right|_{P}}}}{w},
\end{aligned}
$$

with $\xi=\tilde{x}-x^{*}$ and $w=1-\left|x^{*}\right|_{P}$, ensures that $\tilde{\mathcal{E}}_{s}(P)$ is forward invariant and all solutions starting in $\tilde{\mathcal{E}}_{s}(P)$ uniformly exponentially converge to the equilibrium $\tilde{x}=x^{*}$.

Applying Lemma 2 with the selection $x^{*}=-\lambda x_{\circ}$ (and consequently, from (8), $u^{*}=-\lambda u_{\circ}$ ), we obtain that the control law (19) induces forward invariance of $\tilde{\mathcal{E}}_{s}(P)$ and uniform exponential convergence to $x^{*}=-\lambda x_{\circ}$. In particular, since dynamics (16) is linear and $\tilde{x}=x^{*}$ is an equilibrium for the closed loop with (19), then the set (the singleton) $\left\{\tilde{x}=x^{*}\right\}$ is forward invariant and applying, for example, ${ }^{1}$ [7, Prop. 7.5], we obtain local asymptotic stability of $x^{*}$ for dynamics (16), (19) with domain of attraction including $\tilde{\mathcal{E}}_{s}(P)$.

The proof is completed by first noting that, in the original coordinates $x$, the compact set $\left\{\tilde{x}=x^{*}\right\}$ coincides with the origin (this trivially follows from (15a)) and set $\tilde{\mathcal{E}}_{s}(P)$ coincides with set $\mathcal{E}_{a}\left(P, \lambda x_{\circ}\right)$ (as already remarked in (18)). Then, we observe that selection (19) coincides with (9a), (9b), as shown next. In particular, the following relations hold for the quantities in (19b): $\xi=x-\lambda x_{\circ}+\lambda x_{\circ}=x$ and $w=1-\lambda\left|x_{\circ}\right|_{P}$. Then comparing (9b) with (19b) we immediately get $\Psi\left(\tilde{x}, x^{*}\right)=\gamma(x)$.

Moreover, inspired by (9c), we may compute:

$$
\begin{aligned}
\mathrm{dz}_{\sigma}(v) & :=v-\sigma(v)=v-\left(\operatorname{sat}\left(v+\lambda u_{\circ}\right)-\lambda u_{\circ}\right) \\
& =\mathrm{dz}\left(v+\lambda u_{\circ}\right)=\mathrm{dz}\left(\nu+\lambda \delta_{\circ}\right),
\end{aligned}
$$

where we selected $\nu=v+K \lambda x_{\circ}$. Equation (20) may be used to show that $(9 \mathrm{c})$ coincides with (17a) when evaluating (17) with $z=x^{*}+\frac{\tilde{x}-x^{*}}{\Psi\left(\tilde{x}, x^{*}\right)}=\frac{x}{\gamma(x)}-\lambda x_{0}$. Indeed, one gets from (17a) and (20),

$\nu=v+K \lambda x_{\circ}=K\left(\frac{x}{\gamma(x)}-\lambda x_{\circ}\right)+L \mathrm{dz}\left(\nu+\lambda \delta_{\circ}\right)+K \lambda x_{\circ}$.

Finally, we may rearrange (19a) to obtain (9a), by using again (20) and also using (15b) as follows:

$$
\begin{aligned}
u & =\tilde{u}+\lambda u_{\circ}=\gamma(x)\left(\phi\left(x^{*}+\frac{\tilde{x}-x^{*}}{\gamma(x)}\right)-u^{*}\right) \\
& =\gamma(x)\left(\phi\left(-\lambda x_{\circ}+\frac{x}{\gamma(x)}\right)+\lambda u_{\circ}\right) \\
& =\gamma(x)\left(-K \lambda x_{\circ}+\frac{K x}{\gamma(x)}+(L-I) \mathrm{dz}\left(\nu+\lambda \delta_{\circ}\right)+\lambda u_{\circ}\right),
\end{aligned}
$$

\footnotetext{
${ }^{1}$ Note that the cited result [7, Prop. 7.5] is much more general than what we need here. Indeed, the "strong" forward invariance assumed there refers the possibility that solutions be non-unique (whereas here solutions are unique and we simply talk about "forward invariance"). Moreover, the result in [7, Prop. 7.5] is for general hybrid systems, whereas we use it here in the special case of purely continuous-time systems.
}

which coincides with (9a), thus completing the proof.

Remark 2: Replacing Assumption 2 with $A-I$ being invertible, discrete-time extensions of our results can be derived. to this end, the LMI-based design (17) should be revised using [14], while Lemma 2 is valid in both continuous and discrete time [4].

We emphasize that with $\lambda=0$, one gets $\gamma(x) \equiv 1$ from (9b). Then feedback (1), (9) coincides with the symmetric solution (1), (2). Indeed, the selection of $\nu$ in (9c) coincides with the selection of $u$ in (2) (called $u_{s}$ hereafter, to distinguish it from $u$ in (9a)). As a consequence using $\nu=u_{s}$, we get from $(9 a)$

$$
u=K x+L \mathrm{dz}\left(u_{s}\right)-\mathrm{dz}\left(u_{s}\right)=\operatorname{sat}\left(u_{s}\right),
$$

where we used $\operatorname{sat}\left(u_{s}\right)=u_{s}-\mathrm{dz}\left(u_{s}\right)$.

Moreover, $\lambda=0$ implies $\mathcal{E}_{a}\left(P, \lambda x_{\circ}\right)=\mathcal{E}_{a}(P, 0)$ (see again (8)), which means that, by comparing (6) with (13), in this special case $\mathcal{E}_{a}\left(P, \lambda x_{\circ}\right)=\mathcal{E}_{s}(P)$. Then, the statement of Theorem 2 coincides with that of Theorem 1 .

In the general case when $u_{k}^{+} \neq u_{k}^{-}$for all $k$, we can prove that Theorem 2 provides an estimate $\mathcal{E}_{a}\left(P, \lambda x_{\circ}\right)$ of the domain of attraction whose size is always larger than the size of estimate $\mathcal{E}_{s}(P)$ given by Theorem 1 , as stated next.

Proposition 1: If $u_{k}^{+} \neq u_{k}^{-}$for all $k=1, \ldots, m$, then the optimal values $J_{1}^{*}:=\log \operatorname{det}\left(Q_{1}^{*}\right)$ and $J_{2}^{*}:=\log \operatorname{det}\left(Q_{2}^{*}\right)$ of the convex optimizations in Theorems 1 and 2, respectively, satisfy $J_{1}^{*}<J_{2}^{*}$.

Moreover, using $\eta:=\min _{k} \frac{\bar{u}_{k}^{2}}{u_{k}^{2}}>1$, if an optimal solution $(Q, Y, W, U, X)=\left(Q_{1}^{*}, Y_{1}^{*}, \underline{W}_{1}^{*}, U_{1}^{*}, X_{1}^{*}\right)$ from Theorem 1 satisfies $x_{\circ}^{T}\left(Q_{1}^{*}\right)^{-1} x_{\circ}<\eta$, then

$\left(Q_{2}^{*}, Y_{2}^{*}, W_{2}^{*}, U_{2}^{*}, X_{2}^{*}, \mu^{*}\right)=\left(\eta Q_{1}^{*}, \eta Y_{1}^{*}, \eta W_{1}^{*}, \eta U_{1}^{*}, \eta X_{1}^{*}, 1\right)$

is a feasible solution to the constraints of Theorem 2 .

Remark 3: In addition to stating that Theorem 2 always provides better estimates than Theorem 1, Proposition 1 states that, whenever $x_{\circ}^{T}\left(Q_{1}^{*}\right)^{-1} x_{\circ}<\eta$ (namely $x_{\circ}$ falls into the corresponding ellipsoidal estimate scaled by $\eta$ ), then Theorem 2 provides an ellipsoidal estimate that is at least $\eta$ times larger than the one of Theorem 1. That estimate, moreover, is obtained with the same gains $K$ and $L$ as those of Theorem 1, because $\eta$ cancels out in the two left equations of (5). We also emphasize that if $u$ is scalar, then the feasible solution $\left(Q_{2}^{*}, Y_{2}^{*}, W_{2}^{*}, U_{2}^{*}, X_{2}^{*}, \mu^{*}\right)$ characterized in Proposition 1 is also optimal.

Proof: Consider an optimal solution $\left(Q_{1}^{*}, Y_{1}^{*}, W_{1}^{*}, U_{1}^{*}, X_{1}^{*}\right)$ from Theorem 1. If $u_{k}^{+} \neq u_{k}^{-}$for all $k$, then from (7b), also $\left|u_{\circ, k}\right|>0$ for all $k$. As a consequence, selecting $(Q, Y, W, U, X)=\left(Q_{1}^{*}, Y_{1}^{*}, W_{1}^{*}, U_{1}^{*}, X_{1}^{*}\right)$ in the conditions (4a)-(4c), (12) of Theorem 2, there exists a small enough $\mu_{0}>0$ satisfying the left inequality of (12) with $Q=\left(1+\mu_{0}\right) Q_{1}^{*}$, and introducing an increase on each one of the $(1,1)$ terms of the right inequalities of (12) by at least a factor $\eta_{0}:=\min \left\{1+\mu_{0}, \min _{k} \frac{\underline{u}_{k}^{2}+\mu\left|u_{\circ, k}\right|\left(2 \underline{u}_{k}+\left|u_{\circ, k}\right|\right)}{\underline{u}_{k}^{2}}\right\}>1$. Since the remaining inequalities in $(4 b),(4 c)$ are homogeneous in the decision variables, a feasible solution to (4a)-(4c), 
(12) of Theorem 2 is then $\left(Q_{2}, Y_{2}, W_{2}, U_{2}, X_{2}, \mu\right)=$ $\left(\eta_{0} Q_{1}^{*}, \eta_{0} Y_{1}^{*}, \eta_{0} W_{1}^{*}, \eta_{0} U_{1}^{*}, \eta_{0} X_{1}^{*}, \mu_{0}\right)$, for which it is evident that $\log \operatorname{det}\left(Q_{2}\right)>\log \operatorname{det}\left(Q_{1}^{*}\right)$.

The proof of the second statement of the proposition is similar because, with $\mu=1$, entry $(1,1)$ of the right inequality in (12) becomes $\underline{u}_{k}^{2}+2\left|u_{\circ, k}\right| \underline{u}_{k}+\left|u_{\circ, k}\right|^{2}=$ $\left(\underline{u}_{k}+\left|u_{\circ, k}\right|\right)^{2}=\bar{u}_{k}^{2}$.

\section{Simulation EXAMPLE}

The asymmetric stabilizer proposed in Theorem 2 is here compared with the standard symmetric solution of Theorem 1 on the benchmark example in Section 4.1 of [19]. The plant dynamics is given by (1) with

$$
A=\left[\begin{array}{cc}
0.6 & -0.8 \\
0.8 & 0.6
\end{array}\right], \quad B=\left[\begin{array}{l}
1 \\
3
\end{array}\right]
$$

and the saturation limits are $u^{+}=0.5$ and $u^{-}=1.5$. Unlike [19], the state is supposed to be fully available, therefore we do not compare our results to those of [19], which use an output feedback scheme. It is however clear that exponential instability of (21) imposes constraints on the null controllability region and due to this reason the symmetric stability sets are comparable to those in [19, Fig.1,§.4.1].

Solving the convex optimization problem (4), the symmetric stabilizer of Theorem 1 leads to the certified domain of attraction $\mathcal{E}_{s}\left(P_{1}\right)$ illustrated by the black ellipsoid in Figure 1. Following the indications of Remark 1 we may design $K$ with $L=0$. For this single input example, we obtain the same estimate of the basin of attraction as emphasized at the end of Remark 1. The certified domain of attraction $\mathcal{E}_{a}\left(P_{2}, x_{\circ}\right)$ associated with the asymmetric stabilizer of Theorem 2 is instead shown in Figure 1 by the solid red ellipsoid. As suggested in Remark 3, since $x_{\circ}$ is small enough, the same result is obtained by either optimizing (4a)-(4c), (12) (which gives $\mu^{*}=1$ ) or scaling the solution of Theorem 1 . It can be easily noticed that, as expected, its size is considerably increased in comparison to the one of Theorem 1 .

Remark 4: If $L \neq 0$, the algebraic loop (9c) may yield implementation problems. To address this in our simulations, the following lemma has been derived and used.

Lemma 3: With a scalar input $u \in \mathbb{R}$, the solution of the algebraic loop (9c) is given by the explicit equation

$$
\nu=\frac{K x}{\gamma(x)}+L(I-L)^{-1} \mathrm{dz}\left(\delta_{\circ}+\frac{K x}{\gamma(x)}\right) .
$$

Proof: The proof can be straightforwardly derived by inspection, by comparing (9c) and (22) for values of the input above and below the upper and lower thresholds.

Two solutions starting outside the black ellipsoid and inside the red one are reported at the left of Figure 1, showing that, despite being outside the black guaranteed domain of attraction, the blue initial condition is inside both basins of attraction. At the right of Figure 1 we see that all the proposed constructions induce an initially saturated input. With reference to the designs having $L=0$, Figure 2 shows green shaded areas where the input is saturated for Theorem 1 (left) and Theorem 2 (right). Figure 2 also shows accurate
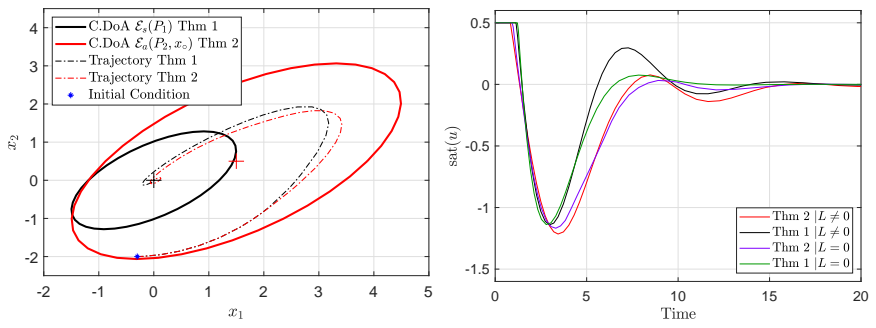

Fig. 1. Left: certified domains of attraction from Theorem 1 (inner black ellipsoid) and Theorem 2 (outer red ellipsoid, centered at $x_{\circ}$ ). Right: time histories of the input sat $(u)$ for the four designs of Theorems 1 and 2, with and without the algebraic loop.
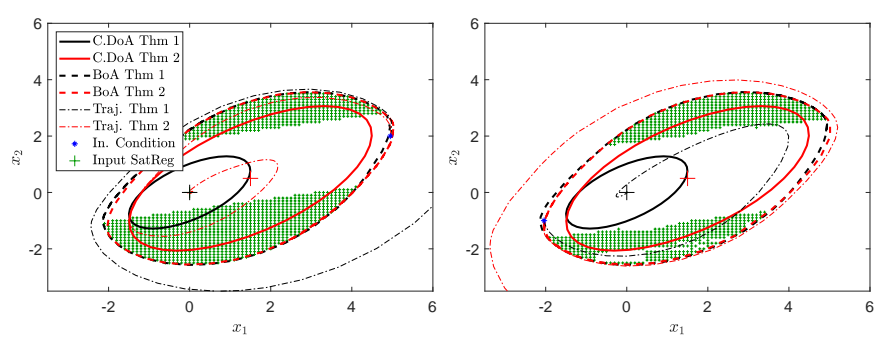

Fig. 2. Actual basins of attraction (BoA) associated with the symmetric design of Theorem 1 (black dashed oval) and the asymmetric design of Theorem 2 (red dashed oval). The quadratic estimates are shown with solid lines. Diverging and converging solutions are also reported in both plots. The green shaded area characterize subsets of the BoA where the symmetric feedback (left) and the asymmetric feedback (right) are saturated.

estimates of the basins of attraction (BoA) induced by each one of the presented stabilizers. The basins of attraction are estimated by picking a set of 50 random initial conditions in the set $\mathcal{E}_{\text {I.C. }}:=\left\{x:\|x\|_{2}=\eta\right\}$, with $\eta=0.01$, by integrating in backward time 50 trajectories and representing them in the time interval $T=\left[t_{\mathrm{in}}, t_{\mathrm{end}}\right]=[80,90]$, where $t_{\mathrm{in}}$ is sufficiently large so that the solutions are indistinguishable from the boundary of the basin of attraction. The correctness of the basins of attraction (BoA) is confirmed by the two sets of trajectories starting just inside/just outside the boundaries and showing converging/diverging behaviour, as expected. Comparing the red and black dashed ovals of Figure 2, we notice similar basins of attraction induced by the two stabilizers. However, from Figure 2 the certified domain of attraction (C.DoA) associated with the asymmetric stabilizer is a significantly less conservative estimate of the actual basin of attraction (red dashed and solid lines), as compared to the symmetric case (black dashed and solid lines). This property may be fundamental in critical applications, where operating within a guaranteed domain of attraction is mandatory.

\section{CONCluding REMARKS}

A nonlinear asymmetric stabilizer has been proposed to guarantee regional stability of a linear system subject to asymmetric input saturation. The proposed paradigm exploits the theory developed in the context of symmetric saturation, by nonlinearly combining a symmetric stabilizer in shifted coordinates with a local stabilizer. The presented numerical example shows that the arising nonlinear controller provides a significant increase of the maximal certified domain of 
attraction, as compared to the classical symmetric approach. Future work includes addressing other performance metrics such as (regional) $\mathcal{L}_{2}$ gains or anti-windup features using asymmetric stabilizers, in addition to addressing the dynamic output feedback design problem.

\section{APPENDIX: PROOF OF LEMMA 2}

We re-state the result in Lemma 2 by converting the notation to that one used in [4], corresponding to the following substitutions: $x^{*} \rightarrow \bar{x}, u^{*} \rightarrow \bar{u}, \tilde{x} \rightarrow x, \xi \rightarrow y$.

Lemma 4: [4] Consider plant $\dot{x}=A x+B \sigma$ and any point $\bar{x} \neq 0$ satisfying $A \bar{x}+B \bar{u}=0$ and $|\bar{x}|_{P}<1$ for some suitable vector $\bar{u}$ and some positive definite matrix $P$. Assume furthermore that a continuous feedback $\sigma=\phi(x)$ is such that function $x^{T} P x$ strictly decreases along solutions in the donut-shaped set $\left\{x: x^{T} P x \in\left[|\bar{x}|_{P}, 1\right]\right\}$.

Then, defining $y:=x-\bar{x}$ and $w:=1-|\bar{x}|_{P}$, the input selection

$$
\begin{aligned}
& \sigma=\bar{u}+\Psi(x, \bar{x})\left(\phi\left(\bar{x}+\frac{x-\bar{x}}{\Psi(x, \bar{x})}\right)-\bar{u}\right) \\
& \Psi(x, \bar{x}):=\min \left\{1, \frac{\frac{\bar{x}^{T} P y}{|\bar{x}|_{P}}+\sqrt{\left(\frac{\bar{x}^{T} P y}{|\bar{x}|_{P}}\right)^{2}+w \frac{y^{T} P y}{|\bar{x}|_{P}}}}{w}\right\},
\end{aligned}
$$

ensures that $\mathcal{E}(P, 1):=\left\{x: x^{T} P x \leq 1\right\}$ is forward invariant and all solutions starting in this set uniformly exponentially converge to the equilibrium $x=\bar{x}$.

Sketch of the proof. The proof is a slight adaptation of the proof in [4]. Similar to the construction in [4, Section 3], the function $(x, \bar{x}) \mapsto \Psi(x, \bar{x})$ is a Minkovski functional whose value is zero for $x=\bar{x}$, and it is one on the ellipse $\partial \mathcal{E}\left(P,|\bar{x}|_{P}\right):=\left\{x: x^{T} P x=|\bar{x}|_{P}\right\}$ (which is a reduced version of the boundary $\partial \mathcal{E}(P, 1)$ ). In particular, for each point $x \in \mathcal{E}\left(P,|\bar{x}|_{P}\right)$, we determine the value of $\Psi(x, \bar{x})$ by imposing the following scaling condition (where the arguments of $\Psi$ have been omitted for simplicity):

$$
\left(\bar{x}+\frac{x-\bar{x}}{\Psi}\right)^{T} P\left(\bar{x}+\frac{x-\bar{x}}{\Psi}\right)=|\bar{x}|_{P} .
$$

Multiplying both sides by $\Psi^{2}$ and solving the equation above for $\Psi$, we get two solutions for each $x \neq \bar{x}$. One of them is negative and is disregarded. The second one corresponds to

$$
\Psi=\frac{-\bar{x}^{T} P y-\sqrt{\left(\bar{x}^{T} P y\right)^{2}-\left(\bar{x}^{T} P \bar{x}-|\bar{x}|_{P}\right) y^{T} P y}}{\bar{x}^{T} P \bar{x}-|\bar{x}|_{P}} .
$$

Dividing both terms by $|\bar{x}|_{P} \neq 0$ and rearranging, we get the expression in the statement of the lemma.

Following the proof technique in [4] we obtain that $\Psi$ is a good Lyapunov function within the set $\mathcal{E}\left(P,|\bar{x}|_{P}\right):=\{x$ : $\left.x^{T} P x \leq|\bar{x}|_{P}\right\}$. In particular, by definition of $\Psi$ in (24), for each point $x$ in $\mathcal{E}\left(P,|\bar{x}|_{P}\right) \backslash\{\bar{x}\}$ we have that $\tilde{x}:=\bar{x}+\frac{x-\bar{x}}{\Psi}$ is on the boundary of $\mathcal{E}\left(P,|\bar{x}|_{P}\right)$, and then the stabilizer in (23) is the convex combination of $\bar{u}$ and $\phi(\tilde{x})$. Due to this fact and because 1$)$ the gradient of $\Psi(x, \bar{x})$ is constant along any ray $\bar{x}+\lambda(x-\bar{x})$ intersected with $\mathcal{E}\left(P,|\bar{x}|_{P}\right)$ (see [4, Lemma 4.2 , item (i)]), and 2) the gradient is aligned with that of
$x^{T} P x$ on the ellipsoid $\left\{x: x^{T} P x=|\bar{x}|_{P}\right\}$, the assumption of strict decrease of $x^{T} P x$ on $\left\{x: x^{T} P x=|\bar{x}|_{P}\right\}$ is enough to conclude strict decrease of $\Psi(x, \bar{x})$ in $\mathcal{E}\left(P,|\bar{x}|_{P}\right)$.

In the donut $\left\{x: x^{T} P x \in\left[|\bar{x}|_{P}, 1\right]\right\}$, the second element in the max reported in the definition of $\Psi$ in (23) is larger than one (by construction), and then $\Psi(x, \bar{x})=1$ implying (again from (23)) that $u=\phi(x)$, which is assumed to induce decrease of $x^{T} P x$ in the considered donut.

\section{REFERENCES}

[1] A. Benzaouia. Constrained stabilization: an enlargement technique of positively invariant sets. IMA Journal of Mathematical Control and Information, 22(1):109-118, 2005.

[2] A. Benzaouia and C. Burgat. Existence of non-symmetrical Lyapunov functions for linear systems. International Journal of Systems Science, 20(4):597-607, 1989.

[3] A. Benzaouia, F. Mesquine, and M. Benhayoun. Saturated Control of Linear Systems. Springer, 2017.

[4] F. Blanchini and S. Miani. Any domain of attraction for a linear constrained system is a tracking domain of attraction. SIAM Journal on Control and Optimization, 38(3):971-994, 2000.

[5] J.M. Gomes da Silva Jr and S. Tarbouriech. Anti-windup design with guaranteed regions of stability: an LMI-based approach. IEEE Transactions on Automatic Control, 50(1):106-111, 2005.

[6] D. Dai, T. Hu, A.R. Teel, and L. Zaccarian. Output feedback design for saturated linear plants using deadzone loops. Automatica, 45(12):2917-2924, 2009.

[7] R. Goebel, R.G. Sanfelice, and A.R. Teel. Hybrid Dynamical Systems: modeling, stability, and robustness. Princeton University Press, 2012.

[8] B.L. Groff, J.M. Gomes da Silva Jr, and G. Valmorbida. Regional stability of discrete-time linear systems subject to asymmetric input saturation. IEEE Conf. on Decision and Control, pages 169-174, 2019.

[9] T. Hu and Z. Lin. Control systems with actuator saturation: analysis and design. Springer Science \& Business Media, 2001.

[10] T. Hu, A.N. Pitsillides, and Z. Lin. Null controllability and stabilization of linear systems subject to asymmetric actuator saturation. In IEEE Conf. on Decision and Control, pages 3254-3259, 2000.

[11] T. Hu, A.N. Pitsillides, and Z. Lin. Null controllability and stabilization of linear systems subject to asymmetric actuator saturation. In V. Kapila and K. Grigoriadis, editors, Actuator Saturation Control, chapter 1, pages 47-76. Marcel Dekker, 2002.

[12] T. Hu, A.R. Teel, and L. Zaccarian. Anti-windup synthesis for linear control systems with input saturation: achieving regional, nonlinear performance. Automatica, 44(2):512-519, 2008.

[13] Y. Li and Z. Lin. An asymmetric Lyapunov function for linear systems with asymmetric actuator saturation. International Journal of Robust Nonlinear Control, 21(4):1-17, 2017.

[14] M. Massimetti, L. Zaccarian, T. Hu, and A.R. Teel. Linear discretetime global and regional anti-windup: an LMI approach. International Journal of Control, 82(12):2179-2192, 2009.

[15] E.F. Mulder, M.V. Kothare, and M. Morari. Multivariable anti-windup controller synthesis using linear matrix inequalities. Automatica, 37(9):1407-1416, 2001.

[16] A. Syaichu-Rohman, R.H. Middleton, and M.M. Seron. A multivariable nonlinear algebraic loop as a QP with applications to MPC. In European Control Conference, pages 1-6, Cambridge, UK, 2003.

[17] S. Tarbouriech, G. Garcia, J.M. Gomes da Silva Jr., and I. Queinnec. Stability and stabilization of linear systems with saturating actuators. Springer-Verlag London Ltd., 2011.

[18] F. Todeschini, S. Formentin, G. Panzani, M. Corno, S. Savaresi, and L. Zaccarian. Nonlinear pressure control for BBW systems via dead zone and anti-windup compensation. IEEE Transactions on Control Systems Technology, 24(4):1419-1431, 2016.

[19] C. Yuan and F. Wu. Switching control of linear systems subject to asymmetric actuator saturation. International Journal of Control, 88(1):204-215, 2015.

[20] S. Zabi, I. Queinnec, G. Garcia, and M. Mazerolles. Time-optimal control for the induction phase of anesthesia. In 20th IFAC World Congress, pages 12708-12713, Toulouse, France, 2017.

[21] L. Zaccarian and A.R. Teel. Modern anti-windup synthesis: control augmentation for actuator saturation. Princeton University Press, Princeton (NJ), 2011. 